\title{
Erratum to: The large noncoding hsrew-n transcripts are essential for thermotolerance and remobilization of hnRNPs, HP1 and RNA polymerase II during recovery from heat shock in Drosophila
}

Subhash C. Lakhotia • Moushami Mallik •

Anand K. Singh • Mukulika Ray

Published online: 10 November 2011

(C) Springer-Verlag 2011

Erratum to: Chromosoma

DOI 10.1007/s00412-011-0341-x

The Fig. 10 of the original version of this article unfortunately contained an error. The correct figure is given below:

The online version of the original article can be found at http://dx.doi. org/10.1007/s00412-011-0341-x.

S. C. Lakhotia $(\bowtie) \cdot$ M. Mallik $\cdot$ A. K. Singh $\cdot$ M. Ray

Cytogenetics Laboratory, Department of Zoology, Banaras Hindu

University,

Varanasi 221005, India

e-mail: lakhotia@bhu.ac.in

Present Address:

M. Mallik

Molecular Neurogenetics Laboratory, Max-Planck Institute

for Molecular Biomedicine,

Roentgenstrasse 20,

48149 Muenster, Germany 
Fig. 10 Redistribution of active RNA pol II during recovery from heat shock is severely affected in cells with down- or upregulated levels of hsrw-n transcripts. Confocal optical sections of polytene chromosome spreads from larvae of different genotypes (indicated on top of each column) immunostained for active form of pol II (red fluorescence) following different treatments indicated on the left of each row. DAPIstained chromosomes are shown in white. The boxed area covering the 93D region is enlarged in the inset in each case. The 93D, $87 \mathrm{C}$, and $87 \mathrm{~A}$ sites are marked with white, green, and yellow arrows, respectively, in each case. Scale bar represents $20 \mu \mathrm{m}$

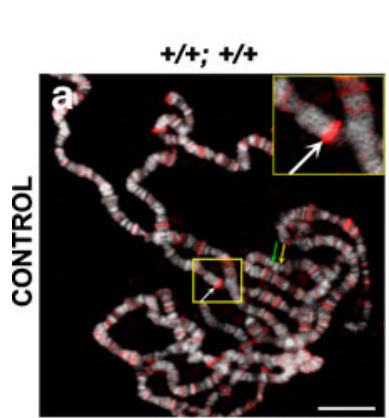

hs-GAL4/CyO;
hsr $\omega-R N A i / h s r \omega-R N A i$

hs-GAL4/CyO;

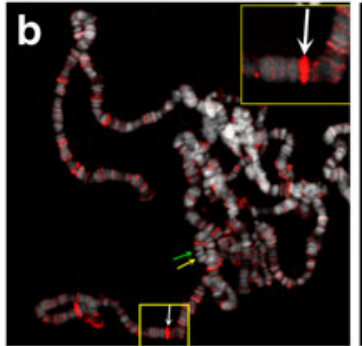

EP93D/EP93D
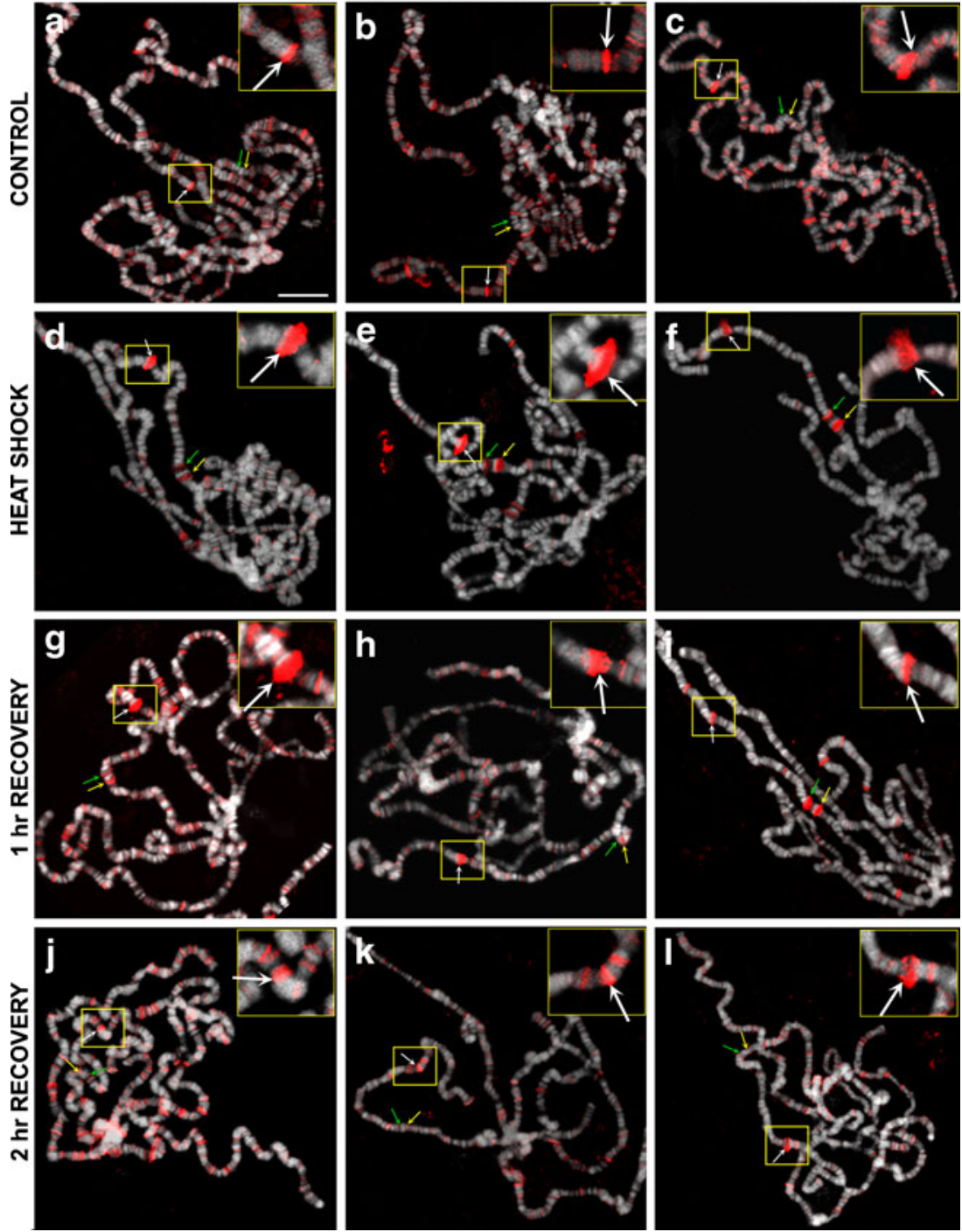